\title{
Precise repair of $m$ Ping excision sites is facilitated by target site duplication derived microhomology
}

David M. Gilbert ${ }^{1}$, M. Catherine Bridges ${ }^{2}$, Ashley E. Strother ${ }^{1}$, Courtney E. Burckhalter ${ }^{1}$, James M. Burnette III and C. Nathan Hancock ${ }^{1 *}$

\begin{abstract}
Background: A key difference between the Tourist and Stowaway families of miniature inverted repeat transposable elements (MITEs) is the manner in which their excision alters the genome. Upon excision, Stowawaylike MITEs and the associated Mariner elements usually leave behind a small duplication and short sequences from the end of the element. These small insertions or deletions known as "footprints" can potentially disrupt coding or regulatory sequences. In contrast, Tourist-like MITEs and the associated PIF/Pong/Harbinger elements generally excise precisely, returning the genome to its original state. The purpose of this study was to determine the mechanisms underlying these excision differences, including the role of the host DNA repair mechanisms.
\end{abstract}

Results: The transposition of the Tourist-like element, mPing, and the Stowaway-like element, 14T32, were evaluated using yeast transposition assays. Assays performed in yeast strains lacking non-homologous end joining (NHEJ) enzymes indicated that the excision sites of both elements were primarily repaired by NHEJ. Altering the target site duplication (TSD) sequences that flank these elements reduced the transposition frequency. Using yeast strains with the ability to repair the excision site by homologous repair showed that some TSD changes disrupt excision of the element. Changing the ends of mPing to produce non-matching TSDs drastically reduced repair of the excision site and resulted in increased generation of footprints.

Conclusions: Together these results indicate that the difference in Tourist and Stowaway excision sites results from transposition mechanism characteristics. The TSDs of both elements play a role in element excision, but only the mPing TSDs actively participate in excision site repair. Our data suggests that Tourist-like elements excise with staggered cleavage of the TSDs, which provides microhomology that facilitates precise repair. This slight modification in the transposition mechanism results in more efficient repair of the double stranded break, and thus, may be less harmful to host genomes by disrupting fewer genes.

Keywords: mPing, Excision site repair, Target site duplication

\section{Background}

Type II DNA transposable elements (TE) are present in most, if not all, eukaryotic genomes, but are especially abundant in plants where they play a role in genome evolution [1]. Plant DNA TEs have been classified into superfamilies including $h A T, M u D R / M U$, CACTA, Mariner, and Harbinger/Pong [2]. Each of these superfamilies is

\footnotetext{
* Correspondence: nathanh@usca.edu

'Department of Biology and Geology, University of South Carolina Aiken, 471 University Parkway, Aiken, SC 29801, USA

Full list of author information is available at the end of the article
}

composed of autonomous elements that encode the proteins required for mobilization and non-autonomous elements that can only be mobilized in trans $[3,4]$. Of special interest are the small ( $<500 \mathrm{bp})$ non-autonomous miniature inverted repeat TEs (MITEs). These are the most abundant TEs in the genome, often reaching thousands of copies, due to their ability for rapid proliferation [5-7]. The two best characterized MITE families, Stowaway and Tourist, have unique characteristics stemming from differences in their transposition mechanisms. Stowawaylike MITEs are mobilized by transposase proteins encoded 
by autonomous Mariner-like elements, produce a $2 \mathrm{bp}$ target site duplication (TSD) upon insertion, and commonly leave small insertions or deletions (footprints) at their excision site [8]. Tourist-like MITEs are mobilized by transposase proteins encoded by the autonomous PIF/Pong-like elements, produce a $3 \mathrm{bp}$ TSD, and generally excise precisely leaving no footprints at their excision site [9].

DNA TEs and their associated MITEs are mobilized by a "cut and paste" mechanism in which transposase proteins bind to the terminal inverted repeats (TIRs), effectively positioning the catalytic domain for the DNA cleavage that is required for both excision and insertion [10]. Staggered cleavage of the genomic DNA at the insertion site results in either a $5^{\prime}$ or $3^{\prime}$ overhang, both of which create small TSDs that flank the inserted elements. Based on the fact that Mariner-like and Stowaway-like elements have 2 bp TSDs, the transposase proteins likely produce a $2 \mathrm{bp}$ overhang upon cleavage of the DNA [8]. The PIF/Pong-like and Tourist-like elements have 3 bp TSDs, indicating cleavage by their encoded transposases produce a $3 \mathrm{bp}$ overhang [11]. Analysis of the excision sites of the elements can elucidate differences in the catalytic mechanism of their specific transposases. For example, the excision sites of the $A c$ and $D s$ elements in both plants and yeast demonstrate that their footprints are palindromic sequences from the flanking DNA, as opposed to pieces of the TE itself [12-14]. This suggests that this transposase cleaves at the end of the element, causing hairpin formation at the ends of the double stranded break. In contrast, the excision sites of Mariner/Stowaway-like elements contain footprints that often include some of the sequences of the element in addition to retaining the TSDs [15]. This indicates that that the Mariner-like transposase cleaves with a staggered cut at the end of the TIR for excision, leaving behind the TSD and a short region of single stranded TIR [15].

Excision of these DNA TEs produces double stranded breaks that are repaired by the host DNA repair mechanisms. This can be accomplished using a complementary template for homologous recombination (HR) or by the non-homologous end joining (NHEJ) pathway [16]. In plants, excision site analysis indicates that many of the repaired sites include insertions or deletions consistent with NHEJ [17-19]. In addition, yeast transposition experiments with the $A c$ element superfamily showed that repair of the double stranded break after excision required NHEJ proteins [12]. This study also showed that microhomology ( $<6 \mathrm{bp}$ ) exposed by end processing between the two strands flanking the element is often used to facilitate repair [12]. Differences in the proteins required for the repair of these ends may hint at the nature of the DNA breaks produced by the different transposases.
The focus of this study was to further characterize the transposition mechanism of the best studied Tourist-like MITE, mPing. In contrast to the previously mentioned elements, mPing excision sites are repaired precisely (leaving no element or TSD sequences). Based on these unique excision sites, we hypothesize that the mPing transposase proteins may cut at the TSD sequences adjacent to the element instead of within the element as seen for Mariner-like elements. Because the TSD sequences are identical, staggered cleavage at this location produces compatible sticky ends, providing microhomology for NHEJ that would easily restore the genome back to its original state before insertion of the element. Based on this hypothesis, we predict that alteration of mPing's TSDs would alter the microhomology and reduce the effectiveness of NHEJ repair. Using a previously developed yeast transposition assay [20, 21], we tested the result of changing the TSDs for a Tourist-like MITE (mPing) and Stowaway-like MITE (OsMar 14T32 or the hyperactive OsMar 14T32-T7). By performing these assays in yeast strains with a defective NHEJ DNA repair pathway, we were able to distinguish between impaired element excision and DNA repair.

\section{Results and discussion}

\section{NHEJ is used for excision site repair}

The yeast transposition assay used for these experiments measures the rate at which the $A D E 2$ gene is repaired in-frame following excision of the TE (Additional file 1) $[14,15,20,21]$. Traditionally, these assays have been performed in haploid yeast lacking an $A D E 2$ homologous template for HR repair of the excision site. Under these conditions the excision site should be repaired only by NHEJ. Performing transposition assays with mPing and $14 T 32$ in haploid yeast strains lacking the NHEJ pathway proteins KU70, MRE11, or RAD50 showed that these proteins are required for efficient repair of the excision sites of both elements (Fig. 1a). Almost no ADE2 revertant colonies were obtained in the $k u 70$ strain, as $K U 70$ is a highly conserved protein involved in the initial binding of the double stranded breaks [22]. For both elements, the rad50 strain showed a higher DNA repair rate than the mre11 strain. This is consistent with a previous study indicating that MRE11 function is more important for repair than RAD50 even though these two proteins function together in the MRX complex to process double stranded breaks before ligation [22, 23]. These results also indicated RAD50 plays a more important role in excision site repair for the $14 T 32$ element than the mPing element [92\% vs. $56 \%$ decrease in repair efficiency (Fig. 1a)]. However, some of this change could be due to a difference in the amount of repair products that result in reading frame disruption. Analysis of excision sites produced in the rad50 background showed that the mPing excision 


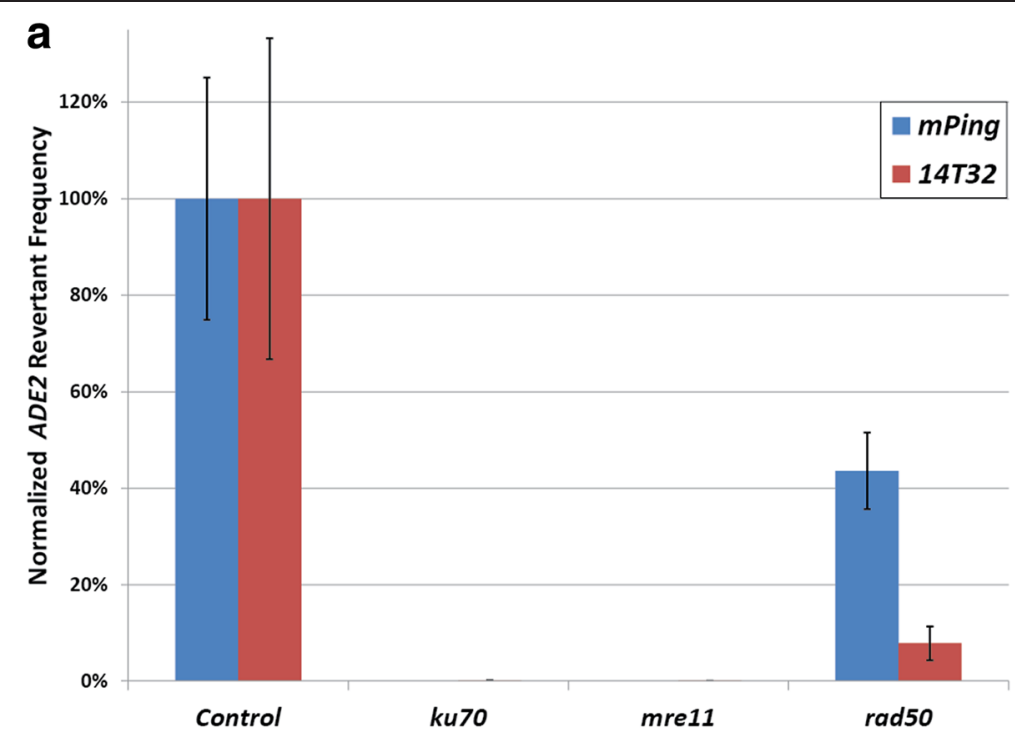

\section{b Excision site analysis}

\section{$A D E 2$}

TAAACCGTTAACAGACC

mPing control

TAAACCGTtaaCAGACC $\quad 96 / 96$

mPing rad50

TAAACCGTtaaCAGACC

\section{$14 T 32$ control}

$\begin{array}{lrl}\text { TAAACCGTTtactccggagtaAACAGACC } & 10 / 19 \\ \text { TAAACCGTttac ggagtaAACAGACC } & 6 / 19 \\ \text { TAAACCGTTtactccg } & \text { taAACAGACC } & 2 / 19 \\ \text { TAAACCGTtacctcggagtaAACAGACC } & 1 / 19 \\ \text { 14T32 rad50 } & & \\ \text { TAAACCG } & \text { taAACAGACC } & 10 / 17 \\ \text { TAAACCGTT } & \text { AACAGACC } & 3 / 17 \\ \text { TAAACC } & \text { AGACC } & 2 / 17 \\ \text { TAA } & \text { AACAGACC } & 1 / 17 \\ \text { TA } & \text { gtaAACAGACC } & 1 / 17\end{array}$

Fig. 1 Transposition assays in NHEJ deficient yeast. Normalized ADE2 revertant frequency for the mPing (blue) and 14T32 (red) elements in control (JIM17) and NHEJ mutant yeast strains (a). Error bars indicate the standard error for 6 replicates. Repaired excision sites from control and rad50 yeast strains (b). Lowercase letters indicate the bases derived from the TSD (mPing) or TIRs and TSDs (14T32)

sites were still repaired precisely, while the $14 T 32$ excision sites had more bases deleted (less precise repair) compared to the control (Fig. 1b). This difference in repair efficiency and quality observed for the two elements in the rad50 strain provides evidence that there are important differences in the nature of the double strand breaks produced by these two elements.
Performing yeast transposition assays in a yeast strain that provides a partial $A D E 2$ template is an effective strategy to evaluate whether HR can be used for excision site repair. This approach has been used to study the $A c$ element ( $h A T$ superfamily, also creates footprints upon excision) where it was reported that when a template is available, about half of the excision sites are repaired by 
HR [12]. In this study, we employed similar methodology to determine if mPing and the hyperactive OsMar 14T32-T7 excision sites are repaired by HR; we used the CB101 yeast strain that contains a partial $A D E 2$ template called $A D E 2^{*}$. This experiment showed that while no significant difference in the rate of $A D E 2$ revertant colonies is observed with or without the $A D E 2 *$ template, CB101 seems to show slightly lower average $A D E 2$ revertants (Fig. 2). This may be due to competition between the two repair pathways or some unknown genetic change present in CB101. This slight difference did not affect our experiments because we were able to normalize within strains. To determine if $\mathrm{HR}$ was occurring in this strain, we analyzed 96 mPing excision sites and fifteen 14T32-T7 excision sites by PCR and digestion with HaeIII (present in $A D E 2 *$ but not in the original $A D E 2)$. Under these conditions, none of the excision sites in either element contained the HaeIII site, and thus, were not repaired by HR at detectable levels (Fig. 2b). This indicates that even when a homologous template is present, the predominant repair pathway for these excision sites is NHEJ.

In order to allow separate analysis of element excision and repair, we developed a yeast strain (DG21B9) that was capable of performing HR at the excision site (contained the $A D E 2^{*}$ template), but also had an impaired NHEJ pathway $(k u 70)$. In this strain, the number of

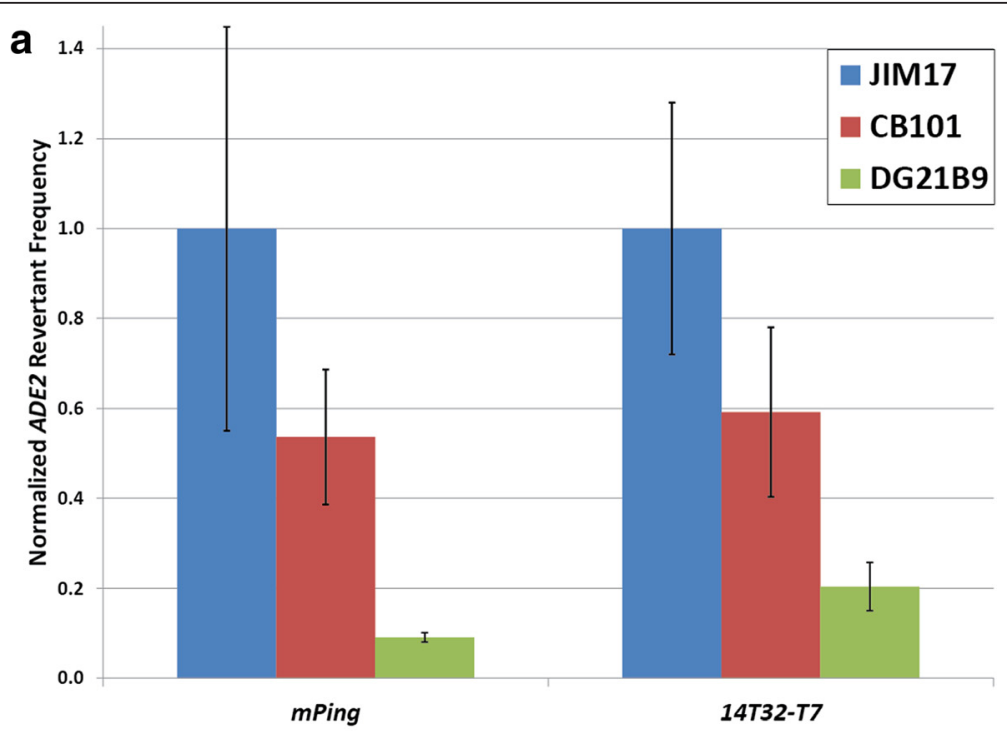

b mPing Excision Sites

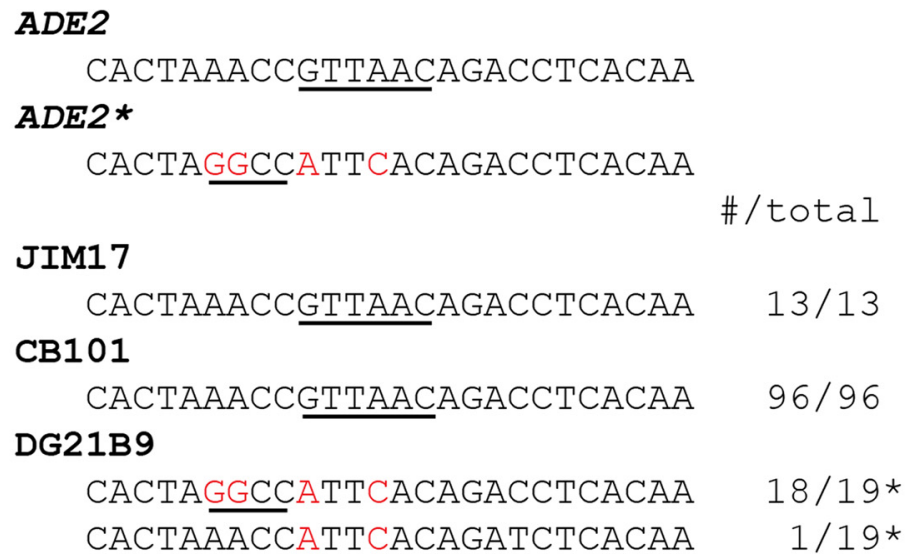

Fig. 2 Transposition assays in yeast with altered DNA repair potentials. ADE2 revertant frequencies for the $m$ Ping and 14T32-T7 elements in yeast strains with different DNA repair mechanisms available for excision site repair (a). JIM17 repairs by NHEJ, CB101 is capable of both HR and NHEJ, and DG21B9 can only repair by HR. Frequencies were normalized to the activity of each transposable element in JIM17. Error bars represent standard error. Sequences identified at the mPing (5' TAA/3' TAA TSDs) excision sites by restriction site analysis and sequencing (b). Underlined sequences indicate the Hpal and Haell sites used for analysis. Red bases are unique to the ADE2* template. *indicates the excision site was repaired by $H R$ using the $A D E 2^{*}$ template 
$A D E 2$ revertant colonies was drastically reduced for both mPing and 14T32-T7 (Fig. 2), but was still higher than observed in the absence of a homologous template (Fig. 1). This drop in activity in this NHEJ deficient strain was consistent with the finding that NHEJ is the dominant pathway for repair of the excision sites. This together with the results for CB101 suggest that HR repair of these breaks functions as a backup to NHEJ and only occurs at about $10-20 \%$ of the rate of NHEJ repair. Analysis of the DG219B ADE2 revertant mPing excision sites by digestion and sequencing showed that $100 \%$ were repaired by HR (Fig. 2). Most of these excision sites (18/19) contained the $A D E 2 *$ specific HaeIII site and the remaining site showed that $A D E 2 *$ was used in such a way as to only remove the $H p a \mathrm{I}$ site and not add the HaeIII site (Fig. 2).

The ability to perform transposition assays in this NHEJ deficient strain (DG21B9) makes it possible to exclude the effects that the quality (i.e. blunt, staggered cut, presence or absence of microhomology) of the DNA break has on repair efficiency. This is because HR is less dependent on the immediate sequence at the end of the double stranded break, instead using sequences farther away from the cleavage site. Thus, this strain provides a method to differentiate whether a mutation affects the rate of NHEJ repair or the rate of excision.

\section{TSD alteration disrupts element excision}

Previous studies have shown that Mariner-like elements require the TSD (TA on both ends) for transposition in vitro [24]. In this study, we confirmed the importance of the conserved TSD for the 14T32-T7 element by changing the TSDs and performing yeast transposition assays. In CB101, changing both bases of the TSDs from TA/TA $\left(5^{\prime} / 3^{\prime}\right)$ to AT/AT almost completely inhibited transposition, while changing just one base (TT/TT or AA/AA) allowed transposition, but at highly reduced rates (Fig. 3a). This experiment was also performed in DG21B9 (HR competent, NHEJ deficient) to confirm that this decrease in activity was due to inhibited excision and not inhibited excision site repair. Figure 3a shows that in DG21B9 alteration of the 14T32-T7 TSDs produced a comparable decrease in activity to the one observed in CB101 yeast. Thus, the drop in activity upon changing the TSDs is likely due to a decrease in excision, and not due to changes in the efficiency of NHEJ. Other researchers have shown that the Mariner-like transposase proteins bind to the TIRs and not the TSDs [25, 26]. Therefore, the TSDs do not likely play a role in binding, but instead play a role in the catalytic mechanism that cleaves the element from the genome.

To determine what role the TSDs play in mPing transposition, we performed yeast assays with mPing elements with altered TSDs. These experiments indicate that alteration of mPing's TSDs also inhibits its transposition (Fig. 3b, Additional file 2a). Based on insertion site analysis, it was already known that $\mathrm{T}$ or A was acceptable at the middle position of the TSD [7]. Changing the middle base to $\mathrm{C}$ or $\mathrm{G}$ (i.e. from TAA/TAA $\left(5^{\prime} / 3^{\prime}\right)$ to TCA/TCA) had a small effect with TGA/TGA TSDs producing more colonies than TCA/TCA TSDs (Additional file 2a). Changing the first base (i.e. GAA/GAA) or third base (i.e. TAC/ TAC) caused a more severe drop in the number of $A D E 2$ revertants. Changing all three bases completely disrupted the transposition of the element (Fig. 3b, Additional file 3a). To determine if this decrease in $A D E 2$ revertants was caused by a drop in excision or from a decreased rate of repair, a subset of these altered elements were tested in the DG21B9 strain (HR only). If altering the TSDs to this extent only affects repair of the excision site and not excision itself, all of these altered TSDs would have the same $A D E 2$ revertant rate as the control in DG21B9. However, almost no $A D E 2$ revertant colonies were detected in the TAC/TAC or GCC/GCC TSD $\left(5^{\prime} / 3^{\prime}\right)$ combinations (Fig. 3b), indicating that these base changes inhibit the ability of the transposase proteins to catalyze excision. It is not clear if this is due to altered enzyme binding or if these bases are directly involved in the catalytic mechanism.

In addition to reducing the number of $A D E 2$ revertant colonies by decreasing excision, sequencing the excision sites indicated that altering the TSDs can result in imprecise repair (Additional file 3b). The production of footprints was especially pronounced for the TAC/TAC TSDs, with 10 of 16 excision sites having indels. The inefficient excision of these altered elements may have resulted in strand cleavage in a non-standard position, creating double stranded breaks that were not as easily repaired.

\section{mPing excision site repair is facilitated by TSD homology}

Based on these initial experiments, we hypothesized that a difference in the double stranded breaks created by the mPing and 14T32 elements results in their excision site differences. Analysis of repaired excision sites shows that Mariner-like transposase proteins produce staggered DNA cleavage within the element, leaving behind some of the TIR sequences (Fig. 1b, Fig. 4) [15]. In contrast, our model for PIF/Pong/Harbinger transposition is that they are mobilized by staggered cleavage of the TSDs, producing three bases of microhomology that facilitates NHEJ (Fig. 4). Based on this, we predicted that changing the TSDs in such a way as to disrupt the microhomology would affect the quality and efficiency of mPing's excision site repair.

The fact that multiple bases are equally acceptable in the middle position of mPing's TSDs allowed experiments to determine if homology between the two TSDs facilitates repair of mPing excision sites. Yeast transposition assays comparing mPing constructs with matching 


\section{a $14 T 32-T 7$}

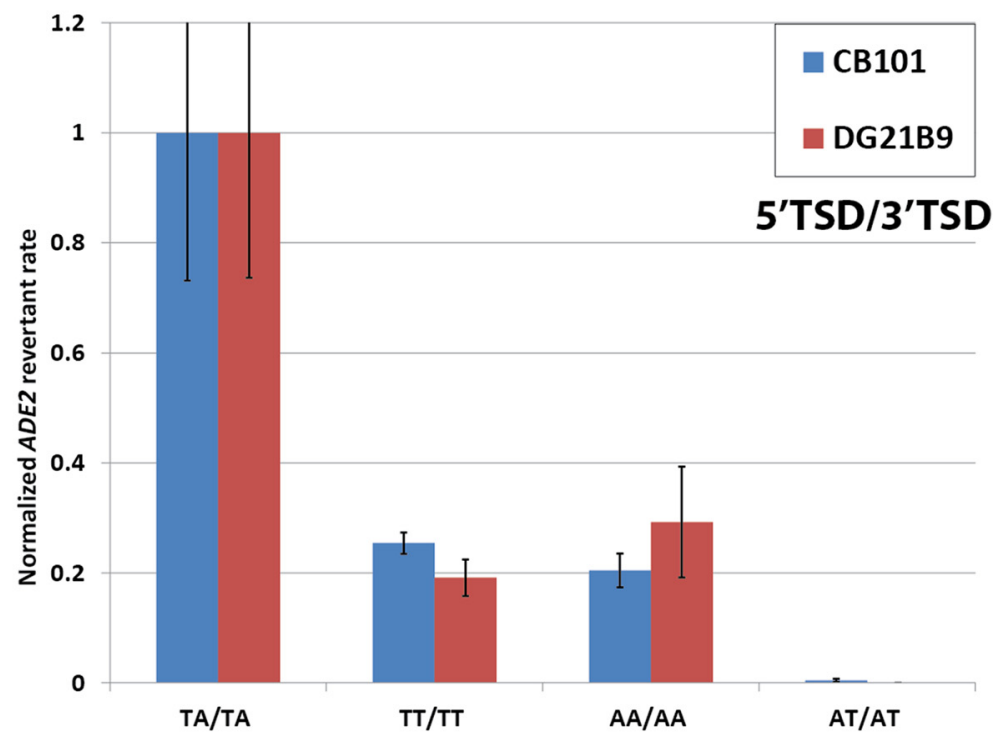

\section{b mPing}

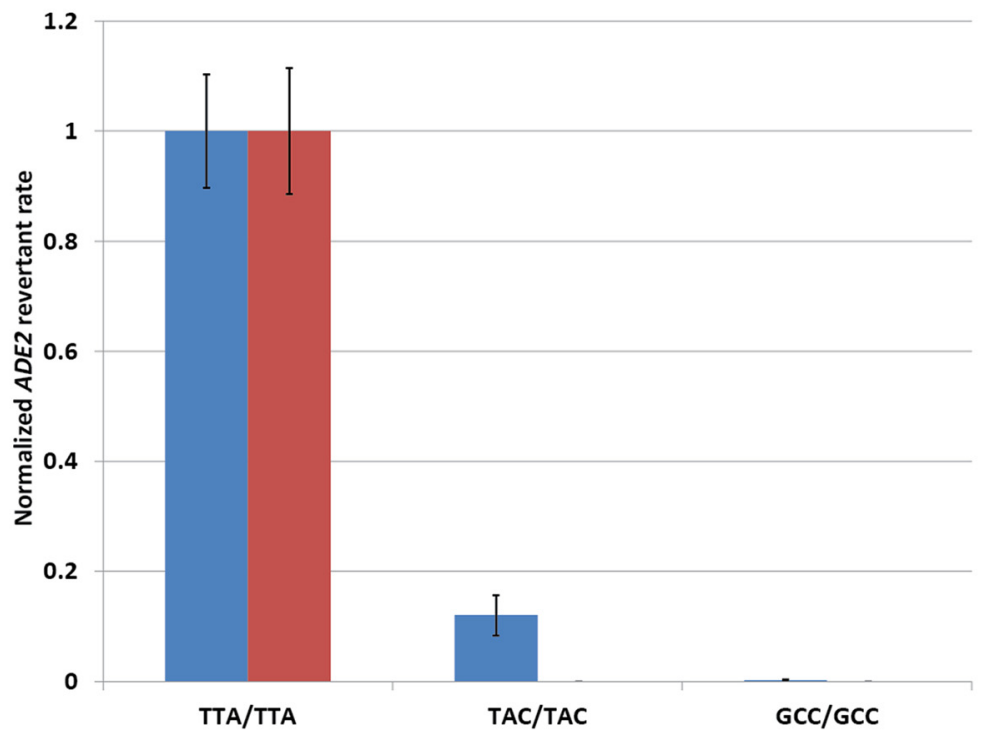

Fig. 3 Transposition assays with altered but matching TSDs. ADE2 revertant rates for 14T32-T7 (a) and mPing (b) elements with altered but matching TSDs. Blue bars indicate the rate in CB101 (capable of both NHEJ and HR), while red bars indicate the rate in DG21B9 (only capable of HR). Values were normalized to the control TSDs (TA/TA for 14T32-T7 and TAA/TAA for mPing) for each yeast strain separately. Error bars represent standard error

TSDs (TTA/TTA and TAA/TAA) and non-matching TSDs (TTA/TAA and TAA/TTA) were performed (Fig. 5, Additional file 3). As shown in CB101 (Fig. 5b) or JIM17 (Additional file 3 ) yeast strains, the mPing elements with non-matching TSDs showed significantly lower transposition than those with matching TSDs. Performing this assay in the DG21B9 strain, which is only capable of repair by HR did not show this effect, with all TSD combinations showing a similar number of $A D E 2$ revertant colonies (Fig. 5b). Together these results indicate that the reduction in $A D E 2$ revertant colonies for nonmatching TSDs is caused by reduced or inaccurate NHEJ repair efficiency. For comparison, similar experiments using the 14T32-T7 element showed that non-matching TSDs produced a similar effect in both NHEJ competent (CB101) and NHEJ deficient (DG21B9) strains (Fig. 5a). This indicates that changing the TSDs of 14T32-T7 only affected its excision and not the repair of the excision site.

Analysis of the excision sites from non-matching TSDs by restriction digest and sequencing was performed to 


\section{a mPing (Tourist-like MITE)}

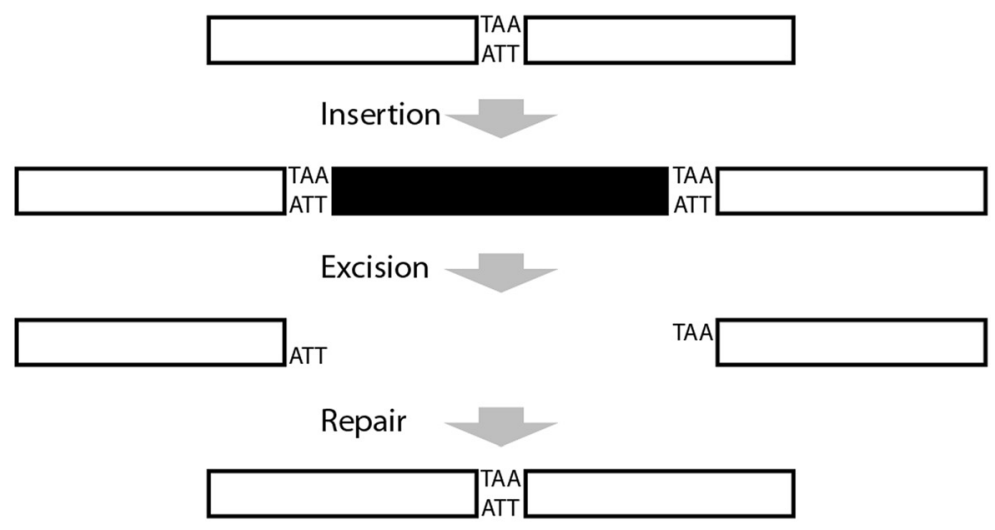

b $14 T 32$ (Stowaway-like MITE)

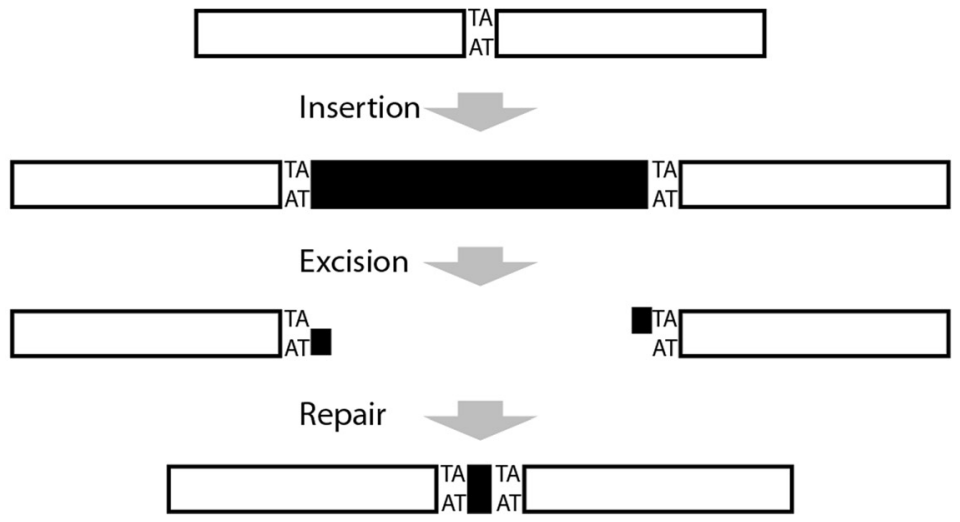

Fig. 4 Model of Tourist-like and Stowaway-like MITE transposition. mPing (a) and 14T32-T7 (b) elements are represented by black boxes, with the TSDs (3 bp and 2 bp respectively) created upon insertion shown as letters. Excision of the mPing element produces TSD derived 5' overhangs that result in precise repair, while 14T32 excision leaves element derived overhangs that results in footprint production

determine how these sites were repaired. Figure 6 shows that in JIM17 and CB101 the excision site produced by an element with TAA/TTA TSDs was repaired fairly precisely with most excision sites only showing one of the TSDs. However, two of the excision sites retained both TSDs, consistent with staggered cleavage at the TSDs that was repaired by NHEJ without microhomology. In contrast, we found that repair of the excision site produced by an element with TTA/TAA TSDs was repaired less precisely in JIM17, or exclusively by HR repair using the $A D E 2^{*}$ template in $\mathrm{CB} 101$ (Fig. 6). This result suggests that the staggered ends created by the TTA/TAA combination were not as easily joined by NHEJ pathway as the TAA/TTA combination. Since a $5^{\prime}$ overhang would create a different set of mismatched bases than a 3 'overhang at the excision site (Table 1), we compared our results to the expected base pairing for each non-matching TSD. Based on this result, we propose that mPing's TSDs cleavage produces a $5^{\prime}$ overhang (Fig. 4, Table 1). A three base 5' overhang would result in the TAA/TTA TSDs forming a
T:T (pyrimidine:pyrimidine) pairing at the middle base of the overhang, a more compatible pairing than the A:A (purine:purine) base paring created by the TTA/TAA TSDs.

Based on this model, we should see that some TSD combinations are more detrimental to excision site repair than others. In fact, analysis of additional combinations of mPing TSDs (TCA and TGA) showed that non-matching TSDs, that according to our model would result in T:C (pyrimidine:pyrimidine) or A:G (purine:purine) mismatches, produced fewer $A D E 2$ revertants than TSD combinations that produce C:A (pyrimidine:purine) and T:G mismatches (pyrimidine:purine) (Additional file 4). Sequence analysis of the excision sites produced by selected TCA and TGA mismatched TSDs (Supplemental 4c) indicates that, for the most part, only one of the TSD sequences is left behind, as is expected of precise repair. However, about $14 \%$ of the time both of the TSDs remained, leaving a footprint. This is in stark contrast to mPing elements with matching TSDs, which have never been observed to leave behind both TSDs upon excision (Fig. 2b) [20, 27]. 


\section{a $14 T 32-T 7$}
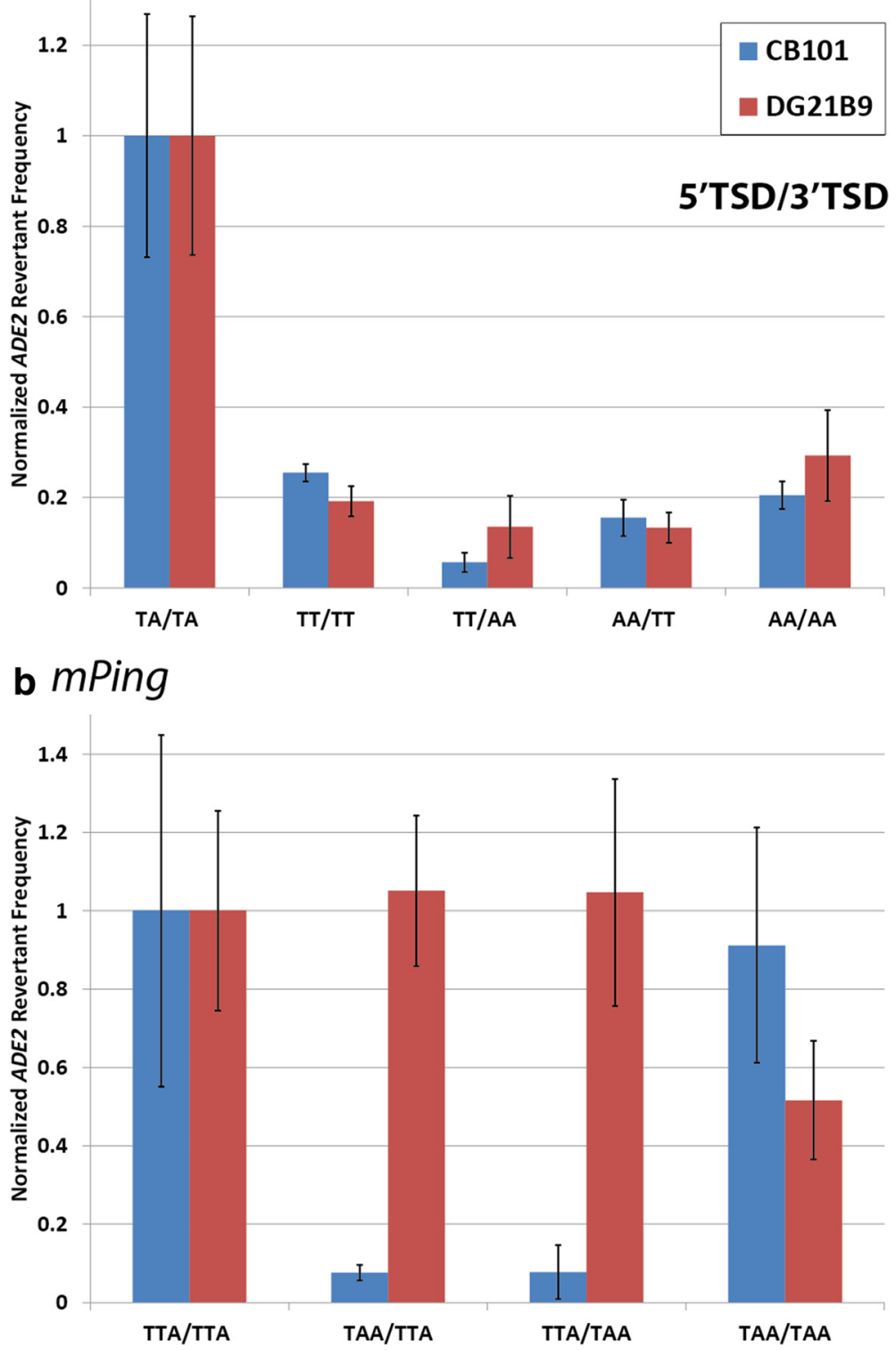

Fig. 5 Transposition assays with non-matching TSDs. Normalized ADE2 revertant frequencies for 14T32-T7 (a) and $m$ Ping (b) elements with altered TSDs. Blue bars indicate the rate in CB101 (capable of both NHEJ and HR), while red bars indicate the rate in DG21B9 (only capable of HR). Values were normalized to the wild-type TSD (left column). Error bars indicate the standard error

It is not clear how common the excision site creation and repair mechanisms observed for mPing are present in other transposon superfamilies. Interestingly, alteration of the P-element TSDs from Drosophila showed a reduction in transposition activity [28]. Also, a recent study with the Os3378 element (Mutator superfamily from rice) that also excises precisely, indicated that alteration of its TSDs reduces the rate of precise excision in yeast [29]. Analysis of these elements in the CB101 and DG21B9 yeast strains would be able to determine if this is due to disruption of excision or excision site repair.
mPing TSDs do not influence target site insertion

Previous research has shown that mPing exhibits a strong preference for insertion into TAA or TTA sequences in the genome $[20,27,30]$. This is consistent with the findings of this study indicating that these sequences are required for efficient excision of the element. However, it was not known if the TSD sequences might play a role in the insertion preference of the element. To address this, 46 insertions of an mPing element with TCA/TCA TSDs were analyzed by sequencing transposon display PCR products [31]. We observed that 45 of the insertions were in TTA or TAA, and only 


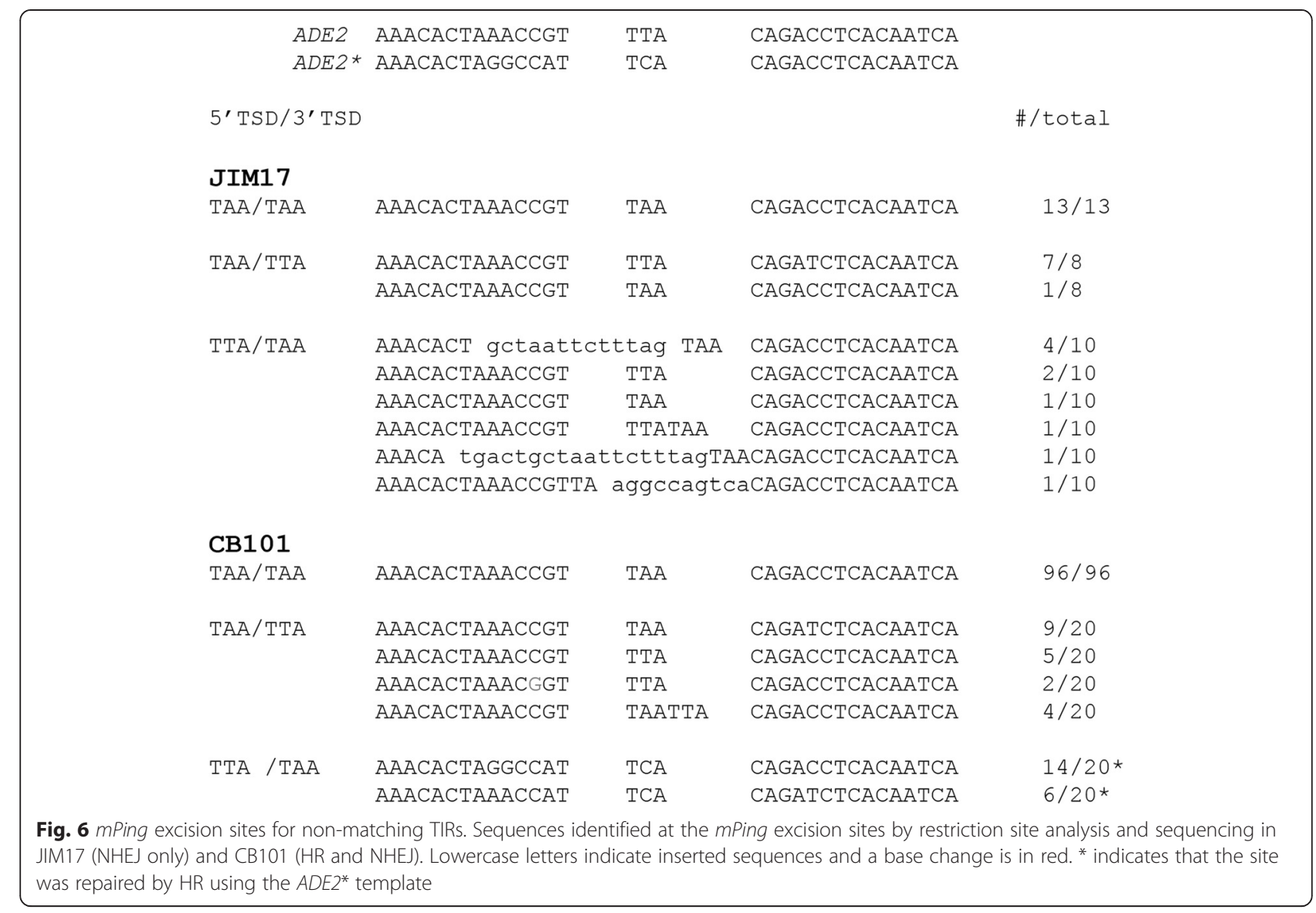

one was in TCA. This is consistent with the results observed for the wild type mPing element [20], suggesting that the TSDs do not play a large role in target site selection.

\section{Conclusions}

These results demonstrate a key difference in the transposition mechanisms used by the Tourist-like and Stowaway-like MITEs. While the excision sites of both mPing and $14 T 32$ elements are primarily repaired by the NHEJ pathway in yeast, the $14 T 32$ element appears to be more sensitive to alteration of NHEJ pathway genes. Our study suggests that the TSDs flanking both elements are required for their efficient excision. On the other hand, complementarity of the two TSDs was found only to be

Table 1 Base pairing that results after $5^{\prime}$ or $3^{\prime}$ staggered cleavage of the mPing TSDs

\begin{tabular}{lll}
\hline \multirow{2}{*}{ mPing target site duplications } & \multicolumn{2}{l}{ Proposed middle base pairing } \\
\cline { 2 - 3 } T' overhang & 3' overhang \\
\hline TAA/TA & A:T & T:A \\
TTA/TAA & A:A & T:T \\
TAA/TA & T:T & A:A \\
TAA/TAA & T:A & A:T \\
\hline
\end{tabular}

critical to the efficiency and precision of mPing's excision site repair. Based on this finding, we conclude that the transposases that excise mPing, and presumably other Tourist-like MITEs, produce a staggered cut at the TSDs that provides microhomology that facilitates precise repair of the excision site.

\section{Methods}

Yeast strains and vectors

\begin{tabular}{|c|c|}
\hline Strain name & Genotype \\
\hline JIM17 & 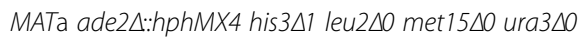 \\
\hline CB101 & 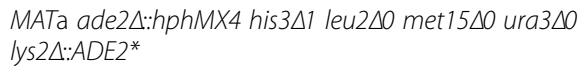 \\
\hline JIM16 & 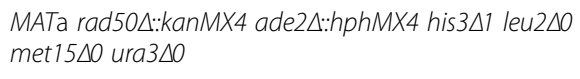 \\
\hline $\mathrm{JIM} 22$ & 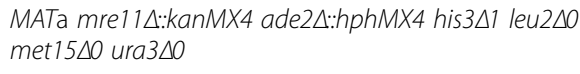 \\
\hline JIM21 & 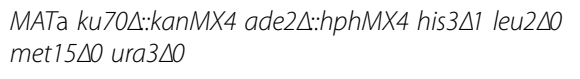 \\
\hline DG21B9 & 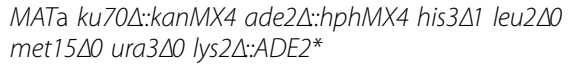 \\
\hline
\end{tabular}


Saccharomyces cerevisiae strains, BY4741 (JIM17) or Yeast Deletion Project strains [32, 33] in the BY4741 background (JIM16, JIM22, JIM21), were adapted for the study by deleting the $A D E 2$ gene using the $h p h M X 4$ (pAG32) cassette replacement technique [34] using the following primers: $A D E 2 h p h M X$ For-CAATCAAGAAAA ACAAGAAAATCGGACAAAACAATCAAGTCCTTGA CAGTCTTGACGTGC, ADE2hphMX Rev-ATAATTATT TGCTGTACAAGTATATCAATAAACTTATATACGCAC TTAACTTCGCATCTG.

The partial $A D E 2$ template $\left(A D E 2^{*}\right)$ was synthesized with the following sequence 5 '-TTTGGCATACGATGG AAGAGGTAACTTCGTTGTAAAGAATAAGGAAATG ATTCCGGAAGCTTTGGAAGTACTGAAGGATCGTC CTTTGTACGCCGAAAAATGGGCACCATTTACTAA AGAATTAGCAGTCATGATTGTGAGATCTGTGAAT GGCCTAGTGTTTTCTTACCCAATTGTAGAGACTA TCCACAAGGACAATATTTGTGACTTATGTTATGC GCCTGCTAGAGTTCCGGACTCCGTTCAACTTAAG GCGAAGTTGTTGGCAGAAAATGCAATCAAATCTTT T-3' and cloned between the BglII and HindIII sites of the pIS 385 disintegrator plasmid [35]. To make the CB101 and DG21B9 yeast strains, this plasmid was then linearized with NruI (New England Biolabs, Massachusetts, USA) and transformed into the LYS2 locus of JIM17 and JIM21, respectively. Selection and screening were performed as described [35] to remove the URA3 selectable marker and identify transformants that maintained the genomic copy of the $A D E 2 *$ template.

The pAG413 Pong ORF1, pAG415 Pong transposase L418A, L420A and pWL89A mPing plasmids were described previously [20]. The pAG415 Osmar14 transposase was made by PCR amplification of the open reading frame from a previously described Osmar14 transposase plasmid [21] with the following primers Osmar 14 For GGGGACAAGTTTGTACAAAAAAGCAGGCTTCATG CAAGAGTACGGCGTGTATGC, Osmar 14 Rev- GGGG ACCACTTTGTACAAGAAAGCTGGGTCTTAAACT GCACTTGGTTGGCTAATGCT. The PCR product was inserted into the Gateway ${ }^{\circ} \mathrm{pDONR}^{\mathrm{m} x} / \mathrm{Zeo}^{\mathrm{P}}$ vector using a BP clonase reaction (Life Technologies, Carlsbad, CA), then transferred into pAG415 GAL ccdb using an LR clonase Reaction (Life Technologies, Carlsbad, CA). The reporter plasmids pwL89A 14 T32 and 14T32-T7 were described previously $[14,21]$. TSD mutations were made to the MITEs mPing and Osmar 14T32-T7 by PCR amplification using primers altered at the TSD (underlined positions indicate TSD), for example:

mPing TGA For - AGTCTCTACAATTGGGTAAGA

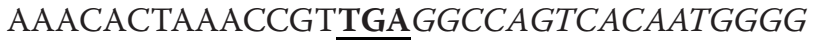
GTTTC

mPing TGA Rev - ACTAAAGAATTAGCAGTCATG ATTGTGAGGTCTGTCAGGCCAGTCACAATGGCTA GTGTC
$14 T 32$ AT For -CTAAAGAATTAGCAGTCATGATT GTGAGGTCTGTTATCTCCCTCCGTCCCAGAAAGAA $G G$, and

$14 T 32$ AT Rev - GTCTCTACAATTGGGTAAGAAAA CACTAAACCGTTATCTCCCTCCGTCCCAGAAAGAAGC

The resulting PCR products were purified using a clean and concentrate kit (Zymo Research, Irvine, CA) and then transformed together with HpaI digested pWL89A using the LiAc method [36]. Mutations were verified by sequencing PCR products or purified plasmids with following primers that flank the $A D E 2 \mathrm{HpaI}$ site: $A D E 2-\mathrm{CF}-\mathrm{GG}$ GTTTTCCATTCGTCTTGAAGTCGAGGAC and ADE2CR-CATTTCCACACCAAATATACCACAACCGGGA.

\section{Yeast transposition assay}

Transposition assays were performed using two techniques depending on the relative transposition rates. For low activity combinations (i.e. Figs. 1, 3 and 4b, and Additional files 3 and 4) transformed yeast were grown in $5 \mathrm{ml}$ of selective media ( $2 \%$ dextrose) at $30{ }^{\circ} \mathrm{C}$ for $48 \mathrm{~h}$, centrifuged to concentrate the culture, plated on selective $2 \%$ galactose plates $(150 \mathrm{~mm})$ lacking adenine, and incubated at $30{ }^{\circ} \mathrm{C}$ for 15 days as described [20]. For experiments with higher rates of transposition (i.e. Fig. 2, 5a and Additional file 1), a $3 \mathrm{ml}$ liquid ( $2 \%$ dextrose) culture was grown for $24 \mathrm{~h}$ at $30{ }^{\circ} \mathrm{C}$ and $100 \mu \mathrm{l}$ was plated on selective $2 \%$ galactose plates $(100 \mathrm{~mm})$ and incubated at $30{ }^{\circ} \mathrm{C}$ for 10 days. A time course of this procedure showed that the number of $A D E 2$ revertant colonies had a linear rate of appearance (Additional file 1). Dilution series of the liquid cultures plated on complete YPD media were used to determine the total number of cells plated. Transposition rate was calculated by dividing the number of $A D E 2$ revertant colonies by the total number of yeast plated.

\section{Excision site analysis}

$A D E 2$ revertant colonies were suspended in $20 \mu \mathrm{l}$ of 1 unit/ $\mu \mathrm{l}$ Zymolyase (Zymo Research, Irvine, CA) and incubated for $15 \mathrm{~min}$ at $37{ }^{\circ} \mathrm{C}$ to lyse the yeast cells. PCR amplification of the excision site was performed using the ADE2-CF and ADE2-CR primers in a $20 \mu \mathrm{l}$ reaction with $2 \mu \mathrm{l}$ of lysed yeast as the template. PCR products were diluted and digested with HpaI or HaeIII (New England Biolabs, Massachusetts, USA) and then analyzed by agarose gel electrophoresis. PCR products were treated with ExoSAP-IT (USB Corporation, Ohio, USA) per instruction of the manufacturer prior to sequencing.

\section{Insertion site analysis}

Transposon display analysis of mPing insertion sites were performed as described previously [20, 30, 31]. Individual bands were sequenced after cutting them from the gel and performing PCR amplification with the transposon display primers. 


\section{Additional files}

Additional file 1: Time course of a yeast transposition assay. ADE2 revertant frequencies for $\mathrm{mPing}$ in the JIM17 strain of yeast. (PDF $305 \mathrm{~kb}$ )

Additional file 2: Additional altered matching TSDs. Additional examples of the of ADE2 revertant frequency for mPing elements with alternative but matching TSDs and their associated excision site sequences. (PDF $360 \mathrm{~kb}$ )

Additional file 3: Non-matching mPing TIRs in JIM17. Chart comparing the frequency of $A D E 2$ revertant colonies produced for $m$ Ping elements with matching (i.e. TAA/TAA) and non-matching TSD sequences (i.e. TAA/TTA) in the JIM17 strain. (PDF 403 kb)

Additional file 4: Additional non-matching mPing TIRs. Charts comparing the frequency of $A D E 2$ revertant colonies produced for $m$ Ping elements with additional combinations of matching (i.e. TCA/TCA) and non-matching TSD sequences (i.e. TAATCA) in the JIM17 strain. (PDF 593 kb)

\section{Abbreviations}

TE: Transposable element; MITE: Miniature inverted repeat transposable element; TSD: Target site duplication; TIR: Terminal inverted repeat; NHEJ: Non-homologous end joining; HR: Homologous recombination.

\section{Competing interests}

The authors declare that they have no competing interests.

\section{Authors' contributions}

$\mathrm{CNH}$ and $\mathrm{JMB}$ developed the experimental design, trained the undergraduates, and drafted the manuscript. DMG, MCB, AES, CEB helped draft and edit the manuscript. Yeast strains were produced by $\mathrm{CNH}, J M B$, MCB, CEB, and DMG. Plasmids were constructed by DMG, CNH, and MCB. Yeast assays and excision site analysis were performed by MCB, AES, and DMG. All authors read and approved the final manuscript.

\section{Authors' information}

The research described in this manuscript was performed almost exclusively as undergraduate research projects. The majority of the work was performed at the University of South Carolina Aiken, a primarily undergraduate institution.

\section{Acknowledgements}

We thank Dr. Guojun Yang for providing the $14 T 32$ and 14T32-T7 constructs. We also thank Dr. Clifford Weil for advice and Christie Bradshaw for technical assistance. We also thank the many undergraduates including Tyler Shealy, Wesley Tindal, Lee B. Sharpe, Lucy Fu, and the spring 2009 HHMI Dynamic Genome class (Justin Brown, Charles B. Allen Jr., Krelin Naidu, Ashley Turner) that helped with aspects of the project. Portions of this research were funded by a grant from the Howard Hughes Medical Institute to Susan R. Wessler. David Gilbert was funded by USCA Connections and USC Magellan grant.

\section{Author details}

'Department of Biology and Geology, University of South Carolina Aiken, 471 University Parkway, Aiken, SC 29801, USA. ${ }^{2}$ Present Address: Department of Pathology and Laboratory Medicine, Medical University of South Carolina, Charleston, SC 29425, USA. ${ }^{3}$ Present Address: College of Natural and Agricultural Sciences, University of California Riverside, Riverside, CA 92521, USA

Received: 29 June 2015 Accepted: 28 August 2015 Published online: 07 September 2015

\section{References}

1. Feschotte C, Jiang N, Wessler SR. Plant transposable elements: Where genetics meets genomics. Nat Rev Genet. 2002;3(5):329-41.

2. Wicker T, Sabot F, Hua-Van A, Bennetzen JL, Capy P, Chalhoub B, et al. A unified classification system for eukaryotic transposable elements. Nat Rev Genet. 2007:8(12):973-82

3. Wessler SR, Bureau TE, White SE. LTR-retrotransposons and MITEs-Important players in the evolution of plant genomes. Curr Opin Genet Dev. 1995;5(6):814-21.
4. Casacuberta J, Santiago N. Plant LTR-retrotransposons and MITEs: control of transposition and impact on the evolution of plant genes and genomes. Gene. 2003;311:1-11

5. Casa AM, Brouwer C, Nagel A, Wang LJ, Zhang Q, Kresovich S, et al. The MITE family Heartbreaker (Hbr): molecular markers in maize. Proc Natl Acad Sci U S A. 2000;97(18):10083-9.

6. Feschotte C, Zhang XY, Wessler SR. Miniature Inverted-repeat Transposable Elements (MITEs) and their relationship with established DNA transposons. In: Craig NL, Craige R, Gellert M, Lambowitz A, editors. Mobile DNA II. Washington D.C.: American Society of Microbiology Press; 2002.

7. Naito K, Cho E, Yang GJ, Campbell MA, Yano K, Okumoto Y, et al. Dramatic amplification of a rice transposable element during recent domestication. Proc Natl Acad Sci U S A. 2006;103(47):17620-5.

8. Plasterk R, Izsvak Z, Ivics Z. Resident aliens-the TC1/Mariner superfamily of transposable elements. Trends Genet. 1999;15(8):326-32.

9. Zhang XY, Jiang N, Feschotte C, Wessler SR. PIF- and Pong-like transposable elements: distribution, evolution and relationship with Tourist-like miniature inverted-repeat transposable elements. Genetics. 2004;166(2):971-86.

10. Yuan YW, Wessler SR. The catalytic domain of all eukaryotic cut-and-paste transposase superfamilies. Proc Natl Acad Sci U S A. 2011;108(19):7884-9.

11. Zhang $X Y$, Feschotte $C$, Zhang Q, Jiang N, Eggleston WB, Wessler SR. P instability factor: an active maize transposon system associated with the amplification of Tourist-like MITEs and a new superfamily of transposases. Proc Natl Acad Sci U S A. 2001;98(22):12572-7.

12. Yu JH, Marshall K, Yamaguchi M, Haber JE, Weil CF. Microhomologydependent end joining and repair of transposon-induced DNA hairpins by host factors in Saccharomyces cerevisiae. Mol Cell Biol. 2004;24(3):1351-64.

13. Rinehart TA, Dean C, Weil CF. Comparative analysis of non-random DNA repair following Ac transposon excision in maize and Arabidopsis. Plant J. 1997;12(6):1419-27.

14. Weil CF, Kunze R. Transposition of maize Ac/Ds transposable elements in the yeast Saccharomyces cerevisiae. Nat Genet. 2000;26(2):187-90.

15. Yang GJ, Weil CF, Wessler SR. A rice TC1/Mariner-like element transposes in yeast. Plant Cell. 2006;18(10):2469-78.

16. Aylon Y, Kupiec M. DSB repair: the yeast paradigm. DNA Repair. 2004:3(8-9):797-815

17. Wessler SR. Phenotypic diversity mediated by the maize transposable elements Ac and Spm. Science. 1988;242(4877):399-405.

18. Scott $L$, LaFoe D, Weil CF. Adjacent sequences influence DNA repair accompanying transposon excision in maize. Genetics. 1996;142(1):237-46.

19. Doseff A, Martienssen R, Sundaresan V. Somatic excision of the Mu1 transposable element of maize. Nucleic Acids Res. 1991;19(3):579-84

20. Hancock C, Zhang F, Wessler S. Transposition of the Tourist-MITE mPing in yeast: an assay that retains key features of catalysis by the Class 2 PIF/ Harbinger superfamily. Mobile DNA. 2010;1(5):5.

21. Yang GJ, Nagel DH, Feschotte C, Hancock CN, Wessler SR. Tuned for transposition: molecular determinants underlying the hyperactivity of a Stowaway MITE. Science. 2009;325(5946):1391-4.

22. Daley JM, Palmbos PL, Wu D, Wilson TE. Nonhomologous end joining in yeast. Annu Rev Genet. 2005;39:431-51.

23. Tsukamoto Y, Kato J, Ikeda H. Budding yeast Rad50, Mre11, Xrs2, and Hdf1, but not Rad52, are involved in the formation of deletions on a dicentric plasmid. Mol Gen Genet. 1997;255(5):543-7.

24. Vos JC, DeBaere I, Plasterk RHA. Transposase is the only nematode protein required for in vitro transposition of TC1. Genes Dev. 1996;10(6):755-61.

25. Zhang L, Dawson A, Finnegan DJ. DNA-binding activity and subunit interaction of the Mariner transposase. Nucleic Acids Res. 2001;29(17):3566-75.

26. Auge-Gouillou C, Hamelin MH, Demattei MV, Periquet G, Bigot Y. The ITR binding domain of the mariner Mos-1 transposase. Mol Genet Genomics. 2001;265(1):58-65.

27. Yang GJ, Zhang F, Hancock CN, Wessler SR. Transposition of the rice miniature inverted repeat transposable element mPing in Arabidopsis thaliana. Proc Natl Acad Sci U S A. 2007;104(26):10962-7.

28. Mullins MC, Rio DC, Rubin GM. cis-acting DNA-sequence requirements for P-element transposition. Genes Dev. 1989:3(5):729-38.

29. Zhao D, Ferguson A, Jiang N. Transposition of a rice Mutator-like element in the yeast Saccharomyces cerevisiae. Plant Cell. 2015;27(1):132-48.

30. Hancock C, Zhang F, Floyd K, Richardson A, LaFayette P, Tucker D, et al. The rice miniature inverted repeat transposable element $m P i n g$ is an effective insertional mutagen in soybean. Plant Physiol. 2011;157(2):552-62. 
31. Jiang N, Bao ZR, Zhang XY, Hirochika H, Eddy SR, McCouch SR, et al. An active DNA transposon family in rice. Nature. 2003;421(6919):163-7.

32. Winzeler EA, Shoemaker DD, Astromoff A, Liang H, Anderson K, Andre B, et al. Functional characterization of the $S$. cerevisiae genome by gene deletion and parallel analysis. Science. 1999;285(5429):901-6.

33. Giaever G, Shoemaker DD, Jones TW, Liang H, Winzeler EA, Astromoff A, et al. Genomic profiling of drug sensitivities via induced haploinsufficiency. Nat Genet. 1999;21(3):278-83.

34. Goldstein AL, McCusker JH. Three new dominant drug resistance cassettes for gene disruption in Saccharomyces cerevisiae. Yeast. 1999;15(14):1541-53.

35. Sadowski I, Su TC, Parent J. Disintegrator vectors for single-copy yeast chromosomal integration. Yeast. 2007;24(5):447-55.

36. Gietz RD, Woods RA. Transformation of yeast by lithium acetate/singlestranded carrier DNA/polyethylene glycol method. Method Enzymol. 2002;350:87-96.

\section{Submit your next manuscript to BioMed Central and take full advantage of:}

- Convenient online submission

- Thorough peer review

- No space constraints or color figure charges

- Immediate publication on acceptance

- Inclusion in PubMed, CAS, Scopus and Google Scholar

- Research which is freely available for redistribution 